\title{
Performance analysis of a two-stage travelling- wave thermo-acoustic engine using Artificial Neural Network
}

\author{
Miniyenkosi Ngcukayitobi ${ }^{1,{ }^{*}}$, Sphumelele Sibutha ${ }^{1,{ }^{* *}}$, Lagouge K. Tartibu ${ }^{1,{ }^{* * *}}$, and Flávio \\ C. Bannwart ${ }^{2, * * * *}$ \\ ${ }^{1}$ Department of Mechanical and Industrial Engineering Technology, University of Johannesburg, \\ Johannesburg, South Africa \\ ${ }^{2}$ School of Mechanical Engineering, University of Campinas, UNICAMP, Campinas, Brazil
}

\begin{abstract}
Thermo-acoustic systems can convert thermal energy into acoustic waves and vice-versa. This conversion is due to the thermo-viscous interaction between the acoustically oscillating gas fluid within a porous medium, referred to as a regenerator, and the pore internal walls. The thermo-acoustic approach is proposed in this study as an alternative sustainable solution for addressing the issue of electricity in remote areas of developing countries. This approach is environmentally friendly as it utilises air as the working medium and therefore does not generate harmful emissions. In this study, a two-stage travelling-wave thermo-acoustic engine has been modelled using DeltaEC. The simulation was performed by considering various input heat for both of the engine stages. The heat input for the first stage was set within the range of 359.48 to $455.75 \mathrm{~W}$, while in the second stage was within the range of 1307.99 to $1656.35 \mathrm{~W}$. Hundred (100) data were generated. This dataset was used to build an Artificial Neural Network (ANN) model. The ANN model was validated using the data extracted from DeltaEC. A good agreement between DeltaEC simulation results and ANN predictions was observed. This study shows that the ANN approach is capable of analysing intricate nonlinear thermoacoustic issues.
\end{abstract}

\section{Introduction}

Waste heat recovery has been identified as one of the promising techniques to further address the issue of energy shortage and environmental pollution [1]. Low-grade heat is produced through a process of fuel combustion or chemical reaction and then dumped into the environment even though it could still play a crucial role in the economy. In this era of industrialization, more attention has been given to the reuse of waste heat generated in

\footnotetext{
* $\quad$ miniyenkosin@ $@$ uj.ac.za

** 217008079@student.uj.ac.za

*** 1tartibu@uj.ac.za

**** fcbannwart@,fem.unicamp.br
} 
different industries and power plants [1]. A bulk of heat energy is dissipated to the atmosphere from many industries together with the hot flue gas emitted from heated products and types of machinery [2]. To improve the energy efficiency of industries, and minimize the production costs, waste heat must be reused [2]. The reduction of such energy losses could contribute to making the whole process more environmentally friendly as heat is regarded as the main factor for the greenhouse effect [2]. The heat energy lost in flue gases cannot be recovered fully and be utilized. However, much of it could be upgraded and recycled by adopting the necessary strategies as described in this research study. The thermo-acoustic approach has been selected to meet the targets of industries due to its simplicity in design, and potential of converting heat power into electric power at a low cost [3].

Thermo-acoustic systems can convert thermal energy into acoustic waves (thermo-acoustic engine) - or mechanical energy - by utilizing what is known as the thermo-acoustic effect. These acoustic waves can be used to generate electric power (thermo-acoustic generator) or to induce cooling (thermo-acoustic refrigerator) [3]. This conversion is due to the thermoviscous interaction between the acoustic particle oscillating along with a gas medium and the pore internal walls of a porous material under sufficiently high-temperature gradient inserted into a resonant waveguide network. The latter is referred to as regenerator for travelling-wave systems, and as stack for the case of standing-wave ones [3]. Travelling sound-wave consists of the propagation of oscillatory displacement of subsequent acoustic particles accompanied by oscillating temperature and pressure. When the acoustic pressure propagates along a narrow channel, oscillating heat transfer between the surface of channel walls and the acoustic particles is enhanced, in both directions, as long as the power intensities involved are high enough. Hence, the aggregation of the oscillations may produce refrigeration due to the thermo-acoustic effect, or, reciprocally, may be generated by the thermo-acoustic effect promoted by a sufficiently high thermal gradient imposed in the direction of propagation [3]. A two-stage travelling-wave thermo-acoustic generator (or engine) is depicted in Fig. 1. It has the following main components: porous medium (regenerator), heat exchangers (cold and hot heat exchangers), and resonance tube.

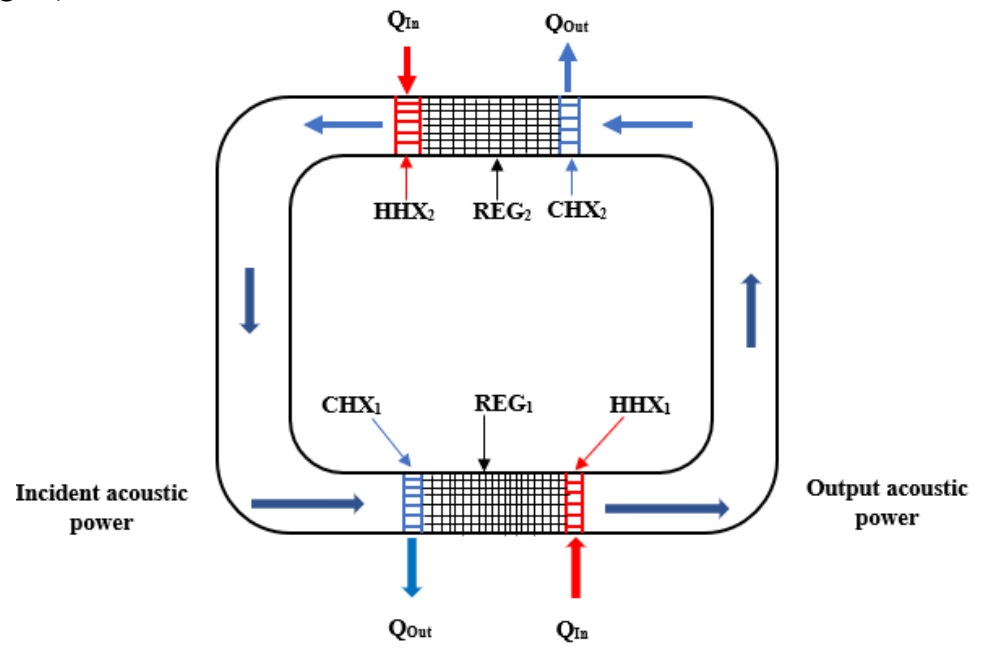

Figure 1: Schematic of a two-stage travelling-wave thermo-acoustic engine.

The porous material (generally sandwiched in-between heat exchangers) are regarded as the main component influencing the efficiency or the performance of the thermo-acoustic systems (refrigerators and engines) [4]. 
The regenerator is defined as the porous material that permits and restricts the working gas to oscillate while in adjacent thermal contact with the solid internal walls. It has many closely spaced surfaces aligned to the length of the resonance tube [4]. The resonance tube defines the frequency of the system, and allows the sound waves to travel through, and also houses the porous medium and heat exchangers. The main function of the two heat exchangers (hot and cold heat exchangers) is to create a temperature gradient along with the porous medium, which is required to raise the acoustic field that is spontaneously generated. In essence, heat exchangers (HX) play a vital role in both cooling and heating processes. They can have various sizes, shapes and can be made of different materials to transfer thermal energy from one liquid to another. In addition, they must have a high overall heat transfer coefficient with low or no acoustic power dissipation [4]. The effective heat transfer rate occurs between the working fluid and narrow-spaced plates of the HX which are aligned in the direction of the wave propagation [5]. The spaces between the plates of HX and the plate size determines the blockage ratio. A smooth flow of the fluid can be attained when the blockage ratios of the porous medium and HX are similar [5]. The working fluid should have a low Prandtl number, high speed of sound, non-flammable, commercially available, and high thermal conductivity (to increase the thermal penetration depth) [5]. Inert gases meet the above-mentioned requirements and thus provide thermo-acoustic systems with the benefits of being environmentally friendly without ozone depletion or global warming issues.

A significant development in the field of thermo-acoustics has been achieved by Kees de Blok [6], who built a device able to convert low-grade heat energy, in the range of $70-200^{\circ} \mathrm{C}$. Multiple regenerator units were proposed [6], to raise the sound power of the thermo-acoustic system at low operating temperature while maintaining higher performance. He also pointed out that the viscous losses can be eliminated by scaling up the regenerator cross-sectional area along the resonance tube. Kees de Blok conducted another experiment on a travellingwave thermo-acoustic engine with a by-pass configuration, and the air was used as the working gas fluid. A threshold equivalent to a temperature gradient of $65 \mathrm{~K}$ was recorded to generate a sound wave within a resonance tube [7]. In a different study, he designed and constructed a four-stage travelling-wave thermo-acoustic generator driven by a temperature gradient of $40 \mathrm{~K}$ across each engine stage [7]. Both of his prototypes are suitable for consuming low-grade waste heat.

Artificial Neural Network (ANN) is defined as the information-processing approach that is based on characteristics similar to biological neural networks [8]. ANN have been developed as a generalization of mathematic models of neural biology, based on the following assumptions:

- The processing of information occurs at the elements called neurons [8].

- Signals are carried through neurons over connection links.

- Each connection link consists of associated weight, which is a typical neural network, multiplies the transmitted signals.

- Each neuron carries an activation function (usually nonlinear) to its network input to determine its output signals [8].

A neural network has a large number of simple processing elements called cells, units, neurons, or nodes. Each neuron is joined to other neurons using directed communication links, each carries associated weight [8]. The weights contain details being used by the network to solve a problem. ANN can be considered in a wide variety of problems, such as recalling data or patterns and storing, performing general mappings from input patterns to output patterns, classifying patterns, grouping similar patterns, or providing solutions to constrained optimization issues [8]. Each neuron is consisting of an internal state, (activation 
level), which is the function of the inputs it has received. Typically, a neuron delivers its activation in a form of signals to various other neurons.

To attain the required correlation between output and input of a network, values must be derived for the activation function and connection weights. The whole process of derivation is named supervised training [8]. ANN are not automated to perform specific work while implemented on the computer. Instead, they are trained to learn patterns used as inputs with respect to the data set. Once the training is completed, new patterns may be displayed to them for classification or prediction [8]. ANN can acquire a knowledge of patterns without direct human control in data from physical models or real systems, computer programs, or other sources. They can deal with many inputs and generate answers that will be suitable for further processing or designers.

ANN have received much attention from researchers worldwide in some engineering fields and thermal science [9]. ANN have been used to solve many intricate issues in air conditioning units, refrigeration, and heat pumps [9]. This application mainly covers the analysis and prediction of the thermal load for air conditioning units and the performance of various refrigeration systems, optimization of operating parameters for refrigeration compressors and thermodynamic performance [9]. Cenk et al [9] applied ANN to heat engineering such as Stirling engines, internal combustion engines, as well as in thermoacoustic research area (specifically for heat engines). The study describes the development of a gasoline engine using the ANN model to predict emissions and performance of the engine. The results reported show that ANN can be considered as a promising flexible tool for classical modelling techniques. Anas et al. [9] applied ANN to thermo-acoustic refrigerators to predict the cooling load in a standing-wave thermo-acoustic refrigerator under given mean pressure and oscillating frequency. The study describes the prediction of the oscillatory heat transfer coefficient in the thermo-acoustic heat exchangers and reports a good agreement between the experimental and ANN results. Yasar et al. [9] also used ANN to predict the power and torque of the Stirling engines as the responses to operating parameters such as charge pressure, engine speed and hot source temperature. The study reports an excellent agreement between experimental and predicted outputs, which confirms that ANN can be used as an alternative intelligent approach for data prediction [9]. Fatih et al [10] conducted a research study on thermo-acoustic instability prediction by joining traditional numerical methods with soft computing tools. The simulation results of the dynamic neural network and black-box approaches with dynamic fuzzy identification show excellent agreement with the results of Comsol (for the burker-Schumann diffusion flame) and Galerkin solver (for coupled thermo-acoustic engine) [10]. The above-mentioned researchers have demonstrated that ANN models are capable of relating the correlation between the output and input parameters, and predict the performance of energy systems [10].

\section{Motivation}

ANN is regarded as a data-driven model, and it acquires knowledge from examples where outputs and inputs are imparted to the network repeatedly and sequentially [11]. ANN can acquire knowledge from examples and that makes it efficient problem-solving with significant benefits in some particular aspects. These aspects are learning and recognizing the underlying relations between outputs and inputs without explicit physical consideration [11]. There has been a significant contribution made by researchers in the development of efficient thermo-acoustic generators [11]. The efficiency of the systems and the nonlinearity associated with the working of the systems remain the major potential area of research. Many existing studies have demonstrated the promising performance of ANN as an alternative 
intelligent tool that can be used to predict future performance in different engineering systems [12].

The driver behind this study is to examine the capability of the ANN model to establish a link between input and output parameters. When sufficient output and input data are provided, the ANN approach can be able to formulate principal correlations between parameters [12]. In this study, a two-stage travelling-wave thermo-acoustic engine has been modelled using DeltaEC. Three (3) parameters affecting the performance of the system have been considered to analyze the system. These parameters include the pressure amplitude, the resonant frequency, the volumetric flow rate, and the input heat. The pressure amplitude and the acoustic power are considered as the main indicators of the performance of the system. The identification of the correlation among the parameters affecting the performance of a thermo-acoustic device and desired performance is the essential foundation of thermoacoustic components design [12].

\section{Detailed description of the proposed approach}

This section discusses the method considered for data collection and the technique used to predict future performance in a thermo-acoustic generator.

\subsection{DeltaEC Modelling}

DeltaEC (Design Environment for Low-amplitude Thermo-acoustic Energy Conversion) has been used by many researchers to build theoretical models and analyze the effect of the geometry configuration of thermo-acoustic systems [13]. This software was developed at Los Alamos National Laboratory by researchers to predict the performance of thermo-acoustic linear systems [13]. It numerically integrates the conservation equations of energy, continuity and momentum. The working principles of the two-stage travelling-wave thermo-acoustic generator are investigated using DeltaEC software, and a code for thermo-acoustic computation is based on the theory of linear thermo-acoustic. It provides a function prototype for characterizing parts in the system such as porous medium, heat exchanger, and resonance tube. Also, the shooting method is adopted to integrate the set boundary conditions and computes the parameters of each segment.

The current study presents the modelling of a two-stage travelling-wave thermo-acoustic system using DeltaEC. Several segments have been selected to develop a travelling-wave looped-tube test rig, as schematically shown in Fig.2.

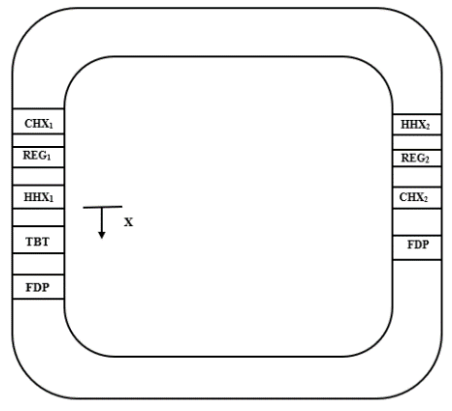

Figure 2: Segments of DeltaEC modelling.

The segments considered include two hot heat exchangers (HHX), two cold heat exchangers (CHX), thermal buffer tube (TBT), feedback pipe (FDP), and two regenerator units. The 
combined elements in the simulated system have a total length of $3.5 \mathrm{~m}$, and each thermoacoustic engine stage was positioned $1.595 \mathrm{~m}$ apart. An operational sound-wave frequency $(f)$ range for the above resonance length is of $98.9 \mathrm{~Hz}$. This value of sound-wave frequency was estimated by using equation 1 and initialized for the thermo-acoustic system [14]. After performing a simulation on DeltaEC, the response frequency (Guess) was adjusted to $97.052 \mathrm{~Hz}$, and this value is very closer to the estimated resonance frequency.

$$
L=\lambda=\frac{c}{f},
$$

with $c$ being the speed of the sound wave (around $346 \mathrm{~m} / \mathrm{s}$ ), $\mathrm{L}$ is the total length of the test rig, and $\lambda$ is the wavelength. The regenerator and both heat exchangers (cold and hot) were modelled as parallel-plate. The model always starts with a "BEGIN" segment, where the sound-wave frequency, mean pressure, temperature, volumetric velocity characteristics, and acoustic pressure characteristic are defined. The initial values at the "BEGIN" segment are unknowns and the target or guess shooting approach was applied. The reverse polish notation (RPN) segments were utilized as targets and guesses; as a result, the test ring ends with several RPN targets that meet final thermo-acoustic properties to put in the appropriate condition properties in the "BEGIN" segment. The second duct of $1.595 \mathrm{~m}$ and the thermoacoustic core of $0.155 \mathrm{~m}$ were added at the end of the first engine stage. The surface temperature for both the cold heat exchanger and hot heat exchangers were set as 1300 and $300 \mathrm{~K}$, respectively. The numerical model has a constant cross-sectional area of $0.008213 \mathrm{~m}^{2}$. Equally, numbered guesses and targets were used for modelling or predicting the performance of the thermo-acoustic system, as required for a deterministic solution. After each execution round, DeltaEC collates the values of guesses to the targets. If the targets do not correspond, the guesses are adjusted and the integration is recurrent. Several simulations were performed for various input heat to model or predict the performance of a thermoacoustic engine. The acoustic power, which is regarded as the main indicator of the performance of the device, was recorded after each successful execution of DeltaEC. The simulation commences at the beginning of the thermal buffer tube (TBT), as indicated by variable $x$ in Fig.2. It computes each segment along the resonance looped tube counterclockwise. The continuous boundary conditions were implemented at $x=0$. The working acoustic pressure amplitude in the thermo-acoustic device was set at $602.28 \mathrm{~Pa}$, the gas inlet temperature at $320 K$, the volumetric flow rate amplitude is $1.8676 \mathrm{e}^{-03} \mathrm{~m}^{3} / \mathrm{s}$ with a phase angle ( $p 1$ and $U 1$ ) of $87.272^{\circ}$; the mean pressure range is $1000 \mathrm{KPa}$ with a phase angle of $0^{\circ}$. The heat input for the first stage was set within the range of 359.48 to $455.75 \mathrm{~W}$, while in the second stage was within the range of 1307.99 to $1656.35 \mathrm{~W}$, and hundred (100) datasets were generated. The frequency, the working pressure amplitude, the volumetric flow rate, the phase angle $\left(p_{l}\right.$ and $\left.U_{l}\right)$, the mean temperature, and the heat supplied to the hot heat exchanger were set as guess parameters. The temperatures in the heat exchangers, the magnitude of the pressure at the final segment of each thermo-acoustic engine stage, the volumetric flow rate at the final segment of each thermo-acoustic engine stage, and the total energy flow at the final segment of both engines were set as the target parameters. The details of the DeltaEC model are shown in Table 1. 
Table 1: Geometrical parameters of the two-stage travelling-wave thermo-acoustic generator.

\begin{tabular}{|l|c|c|}
\hline \multicolumn{1}{|c|}{ Segments } & Diameter (mm) & Length (mm) \\
\hline Ambient heat exchanger & 102.26 & 40 \\
\hline Hot heat exchanger & 102.26 & 20 \\
\hline Regenerator & 102.26 & 95 \\
\hline Duct & 102.26 & 3190 \\
\hline Resonance tube & 102.26 & 3500 \\
\hline
\end{tabular}

\subsection{Determination of samples, training algorithm, and structure for ANN model}

Adequate training sample size and depiction of the training sample for the environment of interest are treated as the first and crucial step in developing of ANN model. An ANN has commonly three layers, namely an output layer, an input layer and hidden layers. Theoretically, there is no restriction on the number of hidden layers. Each of the layers is consisting of a certain number of neurons joined together by the links, which are correlated with weights. The number of output and input neurons depends on the complexity of the modelling problem. In the current study, the input layer has three neurons characterizing the three parameters affecting the performance of the device, and one neuron in the output layer characterizing successively acoustic pressure amplitude or acoustic power.

Several ANN models were built within MATLAB. These models have the same number of input and output layers but different hidden layers. The effect of the number of hidden layers on the model performance was analysed by running several simulations to identify the best ANN architecture. Two architecture are described in this study. Hundred (100) data were extracted from DeltaEC software. These data were divided into three, namely test data set, validation data set, and training data set. The training data set was used to fit the weights of the network or for learning purposes whilst the validation data set was used to reduce overfitting issues that may arise during the training process. The test data was used to evaluate the performance of the trained neural network. The first 70 samples of data $(70 \%)$ were used to train the network, 15 samples of data $(15 \%)$ were used for validation, and the other 15 samples of data $(15 \%)$ was used for testing. The neural fitting app was used to construct and train the network, select data and test its performance using regression analysis and mean square error. A two-layer feed-forward network with linear target neurons and sigmoid hidden neurons was used to fit the data set generated from DeltaEC. The LevenbergMarquardt backpropagation algorithm was utilized to train both of the neural networks. This algorithm was selected simply because it requires less time to process the data. The structures of the neural network depicted in Fig.3, and Fig.4 represents the ANN models adopted in the experiment. The prediction error $(\mathrm{Pe})$, and average prediction error (APe) were estimated using the following equations [15]:

$$
\begin{gathered}
\text { Pe } \%=\left|\frac{\text { Predicted results }- \text { Experimental results }}{\text { Experimental results }}\right|, \\
\mathrm{APe} \%=\frac{\sum_{i-1}^{n}-\text { prediction error } \%}{\mathrm{n}}
\end{gathered}
$$




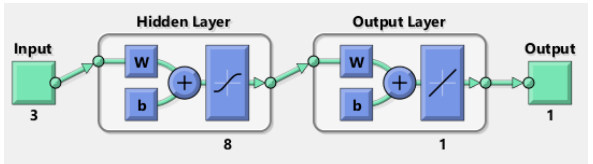

Figure 3: ANN structure (Model 1).

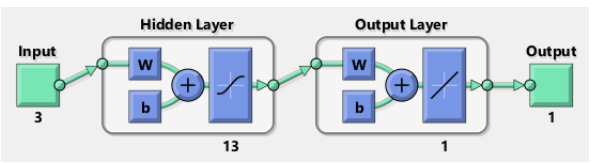

Figure 4: ANN structure (Model 2).

\section{Analysis and results}

\subsection{DeltaEC simulation}

The dataset extracted from DeltaEC software is graphically represented in Fig.5. The results presented in Fig.5 reveals that the heat input to the HHX is directly proportional to the output parameters (pressure amplitude, and acoustic power). Therefore, the rise in the heat supplied to the HHX also causes an increase in the total power as well as in the volumetric flow rate. The acoustic power and pressure amplitude were recorded after each successful execution round, and they both have a similar trend as shown in Fig.5.

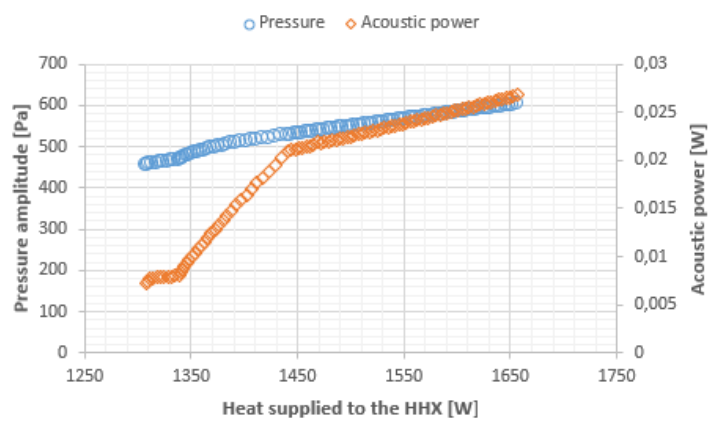

Figure 5: Generated pressure amplitude and acoustic power as a function of input heat.

The simulation was performed by considering various input heat for both of the thermoacoustic engine stages. The input heat for the first engine stage was set within a range of 359.48 to $455.75 \mathrm{~W}$, while in the second stage was within the range of 1307.99 to $1656.35 \mathrm{~W}$. It is also observed that the output parameters (pressure amplitude and acoustic power) were relatively lower at the end of the first stage as compared with the one at the end of the second stage. The addition of the second thermo-acoustic engine stage increases the pressure amplitude as well as the acoustic power output.

\subsection{Analysis of ANN model prediction}

The ANN model predicted acoustic pressure amplitude and acoustic power. Both of the models were trained considering different hidden layers. Three criteria were followed to analyse the performance of the network and hence to reach the optimum one among all proposed networks. 
The first criterion is to reduce network size and complexity if possible. The second criterion is to select the least mean square error (MSE) during the testing phase. The last criterion is to analyse the regression between the corresponding targets (actual output) and network response from the correlation efficient $\left(\mathrm{R}^{2}\right)$ and hence identify the optimum number of hidden neurons. In general, $\mathrm{R}^{2}$ valves varies between positive one and zero, when $\mathrm{R}^{2}$ value closer to positive one indicates an excellent positive linear relationship between the targets and network outputs, while the values near to zero indicate a weak relationship [15]. The above process was performed to identify the architecture of the ANN that yield the lowest discrepancy between the actual and predicted values of the acoustic power and acoustic pressure amplitude successively. The average prediction errors for both of the models between predicted and DeltaEC simulation outputs were computed and plotted again the number of neurons in the hidden layers, as depicted in Fig.6 and Fig.7. It was noted from Fig. 6 that the average prediction error was minimized from eight (8) to fifteen (15) neurons, while the average error was minimized for thirteen (13) neurons (Fig.7). Therefore, the most optimum networks obtained considering the training performance of the pressure amplitude and the acoustic power have respectively eight (8) and thirteen (13) hidden neurons. The pressure amplitude and the acoustic power network must have three input nodes and one output neuron, which can be represented as 3-8-1 and 3-13-1 networks respectively.

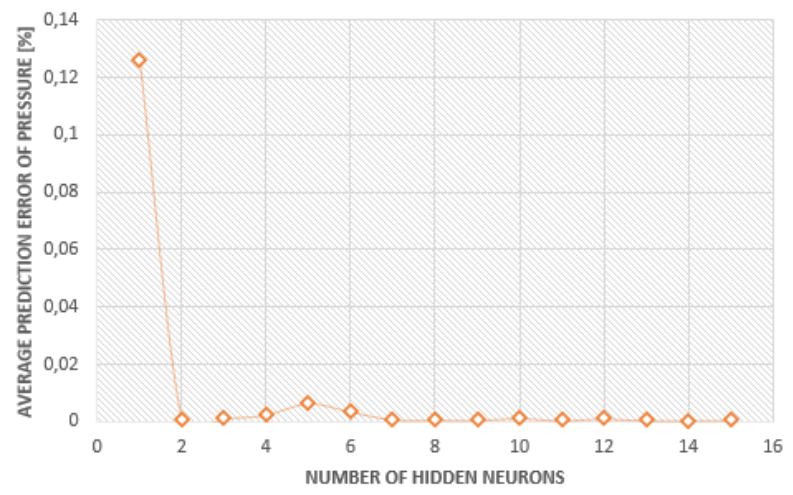

Figure 6: Average prediction error of pressure amplitude [\%] versus the number of hidden neurons.

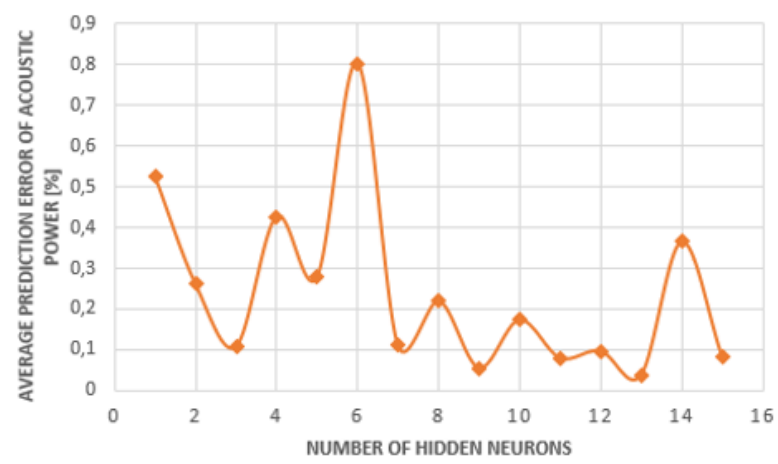

Figure 7: Average prediction error of acoustic power [\%] versus the number of hidden neurons.

It was also observed that the performance of the proposed technique (ANN) in predicting the acoustic power and working pressure amplitude in a two-stage travelling-wave thermo- 
acoustic generator was adequate due to the regression values obtained between target and predicted dataset during validation, training, and testing phases as depicted in Fig.8 and Fig.9.
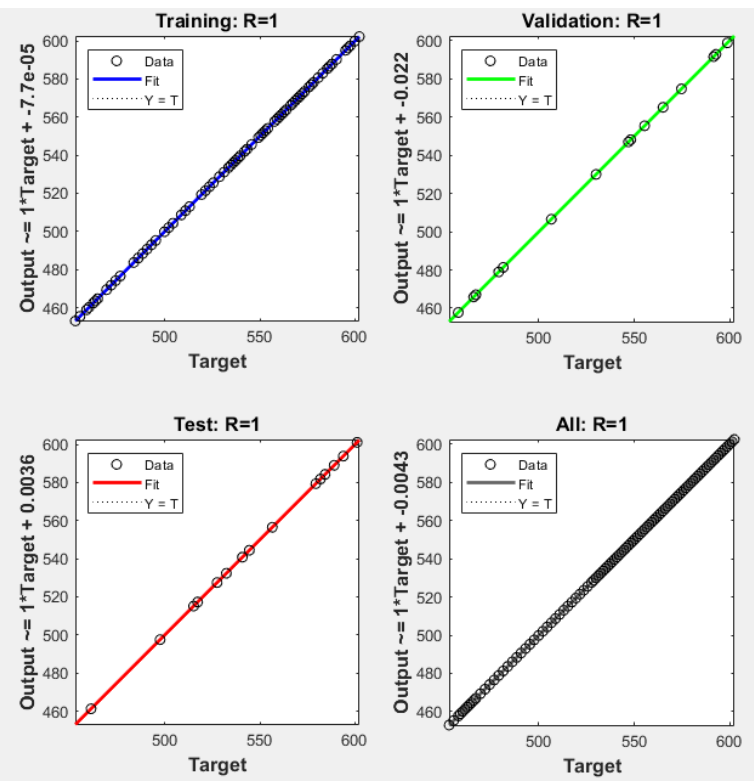

Figure 8: Regression plot for validation, testing, and training phases (pressure amplitude).
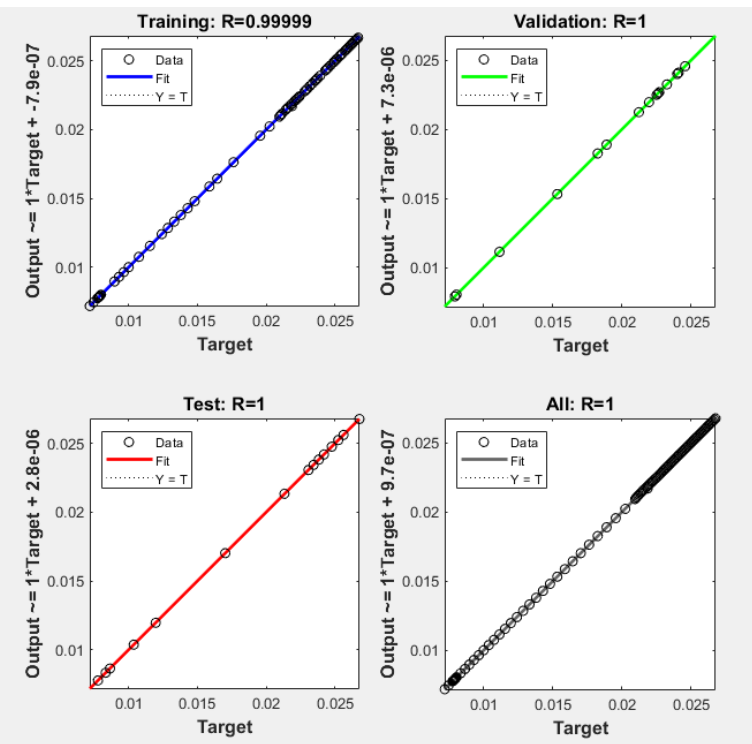

Figure 9: Regression plot for validation, testing, and training phases (acoustic power).

The optimization of the ANN performance was subjected to various constraints such as the construction of the neural network, the amount of both testing and training dataset, and learning parameters. The regression graphs depicted in both Fig. 8 and Fig. 9 indicate the best performance of the neural network in predicting responses of any new dataset input within the range of our research study. To evaluate the prediction ability of our structural neural network, $15 \%$ of the data extracted from DeltaEC simulation were used for the aim of verification. The comparison between the output data extracted from DeltaEC simulation and 
the results predicted from the ANN model was performed and graphically represented in Fig.10, and Fig.11.

Fig.10 shows that "all" the predicted pressure amplitude values, considering the ANN model match closely the targets (DeltaEC output results). This is evidenced by the highest deviation of $2,5 \%$. A similar observation was made, considering the acoustic power. In the latter case, the highest deviation was $0,016 \%$ as shown in Fig. 11 .

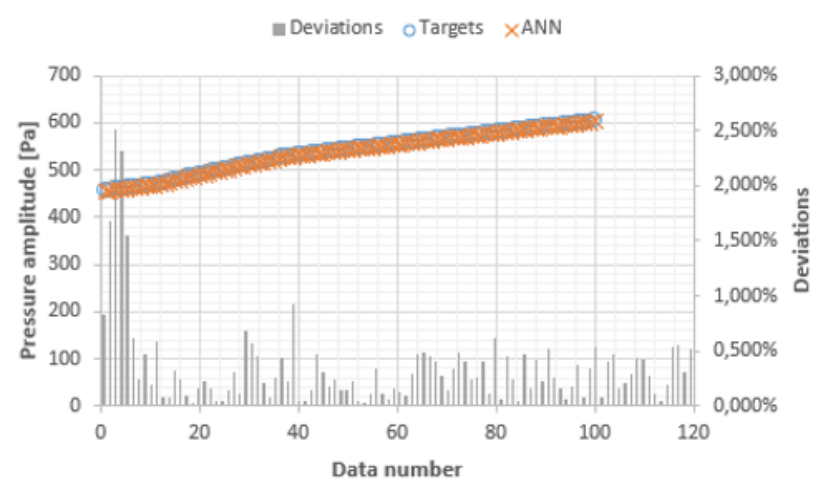

Figure 10: ANN prediction versus output parameters/ pressure amplitude.

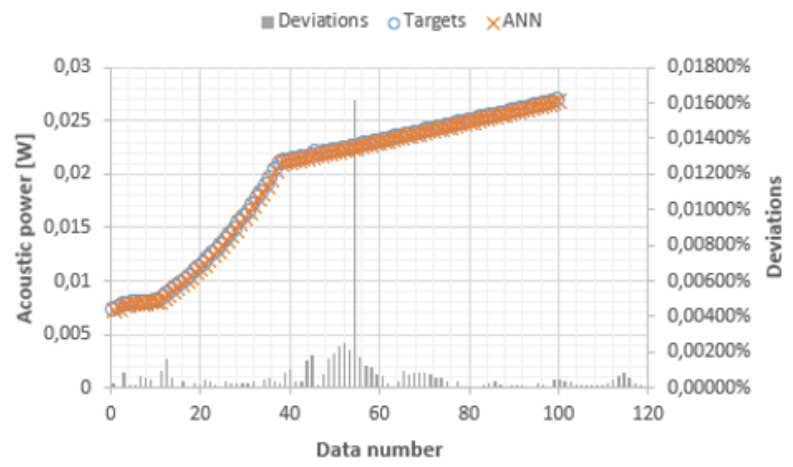

Figure 11: ANN prediction versus output parameters/ acoustic power.

Fig. 10 and Fig.11 show the actual dataset of acoustic power and acoustic pressure amplitude extracted from DeltaEC compared to the ANN predictions. It can be seen that the DeltaEC estimation output results for both of the models have a similar trend to the output results predicted by the ANN model. The agreement between these two results shows the potential for the ANN technique for the prediction of the performance of thermo-acoustic generators. The finding implies that the performance of the proposed ANN models is satisfactory in predicting acoustic power and acoustic pressure amplitude. Also, the ANN approach can be used to predict configurations that were not simulated to improve the analysis of the system.

\section{Conclusions}

This study shows the capabilities of ANN as a flexible modelling technique for estimating the thermo-acoustic output parameters (pressure amplitude and acoustic power) of a two- 
stage travelling-wave thermo-acoustic engine. The process leading to the identification of the best ANN architecture (3-8-1 and 3-13-1) has been described in order to map the correlation between input parameters and responses. In addition, the influence of input parameters on the responses had been analyzed, and it was noted that the heat supplied to the hot heat exchangers has a significant effect on the output parameters. The rise in the heat supplied to the HHX also causes an increase in the acoustic power as well as in the acoustic pressure amplitude.

The present work has shown the significant benefits of ANN to analyse the relationship between parameters describing thermo-acoustic devices and predicts configurations that were not simulated. The approach proposed in this study could potentially improve the analysis of thermo-acoustic systems and provides a better insight into the issue related to their efficiency. This would result in a potential minimization of time-consuming experiments and provide an alternative modelling approach to the thermo-acoustic community of researchers. Future research work must be extended to investigate the ability of ANN to predict experimental data, which are inherently nonlinear.

\section{References}

1. M. Karlsson, M. Abom, M. Lalit, and R. Glav, A note on the applicability of thermoacoustic engines for automotive waste heat recovery. SAE International Journal of materials and manufacturing, vol. 9, issue. 2, pp. 286 - 293 (2016).

2. M.D. Fahel Bin Noor, Ahsan Habib, and Bijoy Mallick, Heat Storage system: A modern way to reuse and recycle Energy to reduce thermal pollution. International conference on mechanical, industrial, and energy engineering (November 2018).

3. A.A.H. Al-Kayiem, Investigation of Travelling-wave Thermoacoustic Engines with Different Configurations, PhD thesis, University of Glasgow (2017).

4. M. Ngcukayitobi, L.K. Tartibu, S.L. Gqibani, Design and construction of a four-stage travelling-wave thermo-acoustic system for power generation. Proceedings of the ASME 2020 International Mechanical Engineering Congress and Exposition, 6, 16-19 (2020).

5. N.M. Hariharan, P. Sivashanmugam, and S. Kasthurirengan, Influence of stack geometry and resonance length on the performance of thermo-acoustic engine. Applied acoustics, vol.73, issue.10, pp. 1052 - 1058, October (2012)

6. Kees de Blok, "Low operating temperature integral thermo acoustic devices for solar cooling and waste heat recovery". Journal of Acoustic Society of America, vol. 123, issue. 5, pp. 3541. DOI: 10.1121/1.2934526 (2008).

7. Kees de Blok, "Novel 4-stage travelling-wave thermo-acoustic power generator". Conference: ASME 2010 3rd Joint US-European Fluids Engineering Summer Meeting collocated with 8th International Conference on Nanochannels, Microchannels, and Minichannels, vol. 7. American Society of Mechanical Engineers; 2010. pp. 73-79

8. Fausett, Fundamentals of neural networks. Architectures, algorithms, and application. Pearson; 1st edition ISBN-10: 0133341860, pp.9-20 (December 19, 1993).

9. A. Rahman, X. Zhang, Prediction of acoustic-wave parameters of thermo-acoustic prime mover through Artificial Neural Network technique: practical approach for thermoacoustics. Journal of thermal science and engineering progress, 8, 257-268 (2018).

10. F. Selimefendigil, H.F. Oztop, Soft Computing methods for thermoacoustic simulation, Numerical Heat Transfer Applications, 66(3):271-288 (2014).

11. S. J. Russell, P. Norvig, Artificial intelligence: a modern approach, 3rd edition. Upper Saddle River, NJ: Prentice-Hall (2010).

12. I.A. Basheer, M. Hajmeer M. Artificial neural networks: fundamentals, computing, design, and application. J Microbiol Meth, vol. 43, issue. 1, pp. 3-31 (2000). 
13. A. Abduljalil, A. Jaworski, Investigation of thermo-acoustic processes in a travellingwave looped-tube thermo-acoustic engine, $\mathrm{PhD}$ thesis, University of Manchester (2012).

14. P. Novotny, S.S. Hsu, A.B. Wang, T. Vit. Investigation of a travelling-wave thermoacoustic engine in a looped-tube. EPJ Web of conferences, pp. 67, 02086 (2014).

15. A. A Rhaman, X. Zhang. Prediction of cooling load for a standing-wave thermo-acoustic refrigerator through Artificial Neural Network technique. $9^{\text {th }}$ International conference on applied energy, ICAE2017. 142, 3780 - 3786 (2017). 